\title{
ESTUDO FLUIDODINÂMICO EM LEITO FIXO DE ADSORÇÃO COMPETITIVA DE ÍONS ZINCO E COBRE EM VERMICULITA EXPANDIDA
}

\author{
H. J. de ALMEIDA ${ }^{1}$, A. F. de ALMEIDA NETO ${ }^{1}$, M. G. A. VIEIRA ${ }^{1}$. \\ ${ }^{1}$ Universidade Estadual de Campinas, Faculdade de Engenharia Química \\ E-mail para contato: melissagav@feq.unicamp
}

\begin{abstract}
RESUMO - Este estudo visa investigar o processo de adsorção binário de íons zinco e cobre em vermiculita expandida, através de sistemas dinâmicos em colunas de leito fixo. Os experimentos foram realizados a diferentes vazões para obter curvas de ruptura e os parâmetros de eficiência: quantidade total (Qt) e útil $(\mathrm{Qu})$ adsorvida dos metais, zona de transferência de massa (ZTM) e porcentagem total de remoção (\%Rem) em leito. Durante o processo adsortivo, a competição entre ambos os metais pelos sítios ativos da vermiculita demonstrou uma maior seletividade dos íons cobre.
\end{abstract}

\section{INTRODUÇÃO}

A quantidade de efluentes industriais gerados anualmente tem crescido de acordo com o desenvolvimento da indústria. Tais efluentes normalmente contêm substâncias tóxicas, que não podem ser descartadas sem o tratamento adequado devido seu alto risco de contaminação do meio ambiente e consequente interferência em ecossistemas e na saúde de organismos vivos (Derísio, 2005). É de grande importância, portanto, o desenvolvimento de políticas de gerenciamento de resíduos e efluentes que evitem ou minimizem a poluição dos recursos hídricos, mitigando, assim, possíveis danos ao meio aquático (Peric e Mendvidovic, 2004).

Metais pesados, como cobre e zinco, são utilizados em larga escala em diversos processos produtivos. Embora sejam encontrados naturalmente, são prejudiciais ao solo, alteram as características físico-químicas da água e acarretam inúmeras patologias a seres vivos em geral, quando descartados em grandes quantidades. A concentração máxima de zinco para lançamento de efluentes nas águas superficiais é de $5 \mathrm{mg} / \mathrm{L}$, enquanto a concentração máxima de cobre é de $1 \mathrm{mg} / \mathrm{L}$ (CONAMA, 2011).

Técnicas de tratamento alternativas, como osmose reversa, adsorção e ultrafiltração, estão sendo empregadas na tentativa de otimizar a remoção e/ou recuperação de íons metálicos (Bhattacharya et al., 2006; Chui, 2004). Com essa perspectiva, a adsorção se mostrou um método de destaque, pois é relativamente simples, versátil e de baixo custo (Almeida Neto, 2011). Entre as argilominerais, a vermiculita possui a maior capacidade de troca catiônica (CTC), com valores registrados na faixa de 100 a $150 \mathrm{meq} / 100 \mathrm{~g}$ (Grim, 1968). Após rápido aquecimento, a vermiculita expande, perdendo moléculas de água e adquirindo propriedades bastante interessantes, como maior CTC, inércia química, leveza e refratariedade (Franco et al., 2010; Machado et al., 2006).

Nishikawa et al. (2012 a), em seu estudo sobre adsorção de soluções simples de $\mathrm{Cu}(\mathrm{II}), \mathrm{Zn}(\mathrm{II}), \mathrm{Pb}$ (II) e $\mathrm{Cr}(\mathrm{II})$ em vermiculita expandida brasileira, identificou o potencial de 
adsorção promissor do material. A vermiculita foi também analisada com o mesmo propósito por Alexandre-Franco et al. (2011), Ugarte e Monte (2006) e Fonseca et al. (2005). Este estudo visa explorar o processo adsortivo binário de íons cobre e zinco em vermiculita expandida brasileira, através de sistema dinâmico de leito fixo em coluna, com diferentes vazões para obtenção de curvas de ruptura e dos parâmetros de eficiência: quantidade total e útil de metal adsorvido, zona de transferência de massa e porcentagem total de remoção.

\section{Material e Métodos}

A vermiculita é uma argila mineral micácea, formada por folhas tetraédricas de $\left(\mathrm{SiO}_{4}\right)^{-4}$ e folhas octaédricas de $\mathrm{Mg}_{3}(\mathrm{OH})_{6}$. Em seus espaços interfoliares, há cátions hidratados que podem ser trocados por outros cátions presente na solução. A vermiculita expandida utilizada nesse estudo foi fornecida pela Brasil Minérios LTDA. Após trituração e peneiramento, obtiveram-se partículas de diâmetro médio $0,855 \mathrm{~mm}$. O adsorvente foi previamente caracterizado por Nishikawa et al. (2012 b).

As soluções dos metais investigados foram preparadas a partir da dissolução dos sais de nitrato de cobre $\left(\mathrm{Cu}\left(\mathrm{NO}_{3}\right)_{2} \cdot 3 \mathrm{H}_{2} \mathrm{O}\right)$ e nitrato de zinco $\left(\mathrm{Zn}\left(\mathrm{NO}_{3}\right)_{2} \cdot 6 \mathrm{H}_{2} \mathrm{O}\right)$ em água desionizada. $\mathrm{O}$ intervalo de intersecção entre as faixas de $\mathrm{pH}$ de ambos metais no qual não ocorre precipitação química foi determinado por especiação metálica fornecida pelos programas HYDRA e MEDUSA, enquanto o controle foi feito pela adição de ácido nítrico.

Os experimentos de adsorção em leito fixo foram realizados, a temperatura ambiente, em colunas de vidro com $15 \mathrm{~cm}$ de comprimento e $1,5 \mathrm{~cm}$ de diâmetro interno. Com auxílio de bomba peristáltica, solução contendo ambos os metais passou de forma ascendente pela coluna preenchida com o material adsorvente. Em tempos pré-determinados, alíquotas da solução tratada foram coletadas por coletor automático. A concentração dos íons metálicos na solução inicial e nas amostras foi determinada no espectrofotômetro de absorção atômico AA7000, da Shimadzu (Japão). Quatro vazões foram utilizadas, com as respectivas concentrações de cobre e zinco na solução inicial: $1 \mathrm{~mL} / \mathrm{min}, 0,7230$ e $0,6764 \mathrm{mmol} / \mathrm{L} ; 3 \mathrm{~mL} / \mathrm{min}, 0,4682$ e 0,5488 $\mathrm{mmol} / \mathrm{L} ; 5 \mathrm{~mL} / \mathrm{min}, 0,5908$ e $0,5227 \mathrm{mmol} / \mathrm{L} ; 7 \mathrm{~mL} / \mathrm{min}, 0,5908$ e 0,5227 $\mathrm{mmol} / \mathrm{L}$.

A fim de analisar o desempenho do leito na adsorção de cada adsorbato em função da vazão escolhida, foram obtidas as curvas de ruptura, que são gráficos da razão entre a concentração em cada tempo de coleta pela concentração inicial $\left(C / \mathrm{C}_{0}\right)$ em função do tempo $(\mathrm{t})$, em minutos. As quantidades total $\left(\mathrm{q}_{\mathrm{t}}\right)$ e útil $\left(\mathrm{q}_{\mathrm{u}}\right)$ de cobre e zinco adsorvidas, em $\mathrm{mmol} / \mathrm{L}$, foram calculadas, respectivamente, pelas Equações 1 e 2, obtidas por balanço de massa no sistema em coluna. A porcentagem total de remoção $\left(\% \mathrm{R}_{\mathrm{L}}\right)$ pôde ser calculada pela divisão da quantidade de metal removido até o ponto de saturação pela quantidade total de íons metálicos presentes na solução inicial. A ZTM foi determinada por meio da Equação 3 (Geankoplis, 1993), na qual é possível perceber a relação de valores menores com maior eficiência do leito.

$$
\begin{aligned}
& q_{t}=\frac{C_{0} \cdot Q}{m} \int_{0}^{\infty}\left(1-\frac{C}{C_{0}}\right) d t \\
& q_{u}=\frac{C_{0} \cdot Q}{m} \int_{0}^{t_{r}}\left(1-\frac{C}{C_{0}}\right) d t
\end{aligned}
$$




$$
Z T M=\left(1-\frac{q_{u}}{q_{t}}\right) \cdot H_{L}
$$

Sendo $\mathrm{Q}$ a vazão da coluna $(\mathrm{mL} / \mathrm{min}), \mathrm{m}$ a massa de argila seca (gramas), $\mathrm{C}_{0}$ a concentração inicial de metal na fase líquida $(\mathrm{mmol} / \mathrm{L}), \mathrm{C}(\mathrm{t})$ a concentração de saída da coluna em cada instante de tempo $(\mathrm{mmol} / \mathrm{L}), \mathrm{t}_{\mathrm{r}}$ o tempo no qual a $\mathrm{C}(\mathrm{t})$ é igual à $5 \%$ de $\mathrm{C}_{0}$ e $\mathrm{H}_{\mathrm{L}}$ é a altura do leito.

\section{RESULTADOS E DISCUSSÃO}

A Figura 1 apresenta os diagramas de especiação metálica com concentração teórica de $0,5 \mathrm{mmol} / \mathrm{L}$ para ambos os metais. Para garantir que não houvesse precipitação química dos íons cobre e zinco com outros componentes do meio, o pH foi mantido abaixo de 4,5, de acordo com os diagramas obtidos. Cabe ressaltar que a especiação considera apenas os componentes da solução, desconsiderando a presença do material adsorvente.

Figura 1 - Especiação metálica em sistema bicomposto de (a) cobre e (b) zinco.

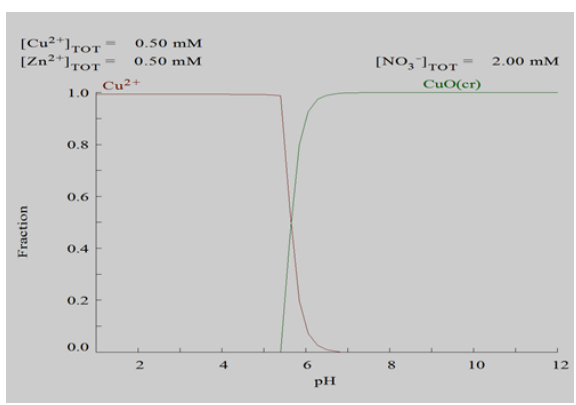

(a)

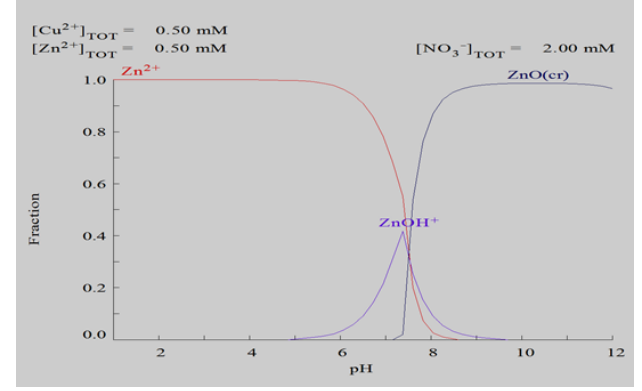

(b)

As curvas de ruptura correspondente ao processo de adsorção em sistema dinâmico de leito fixo podem ser observadas na Figura 2. A exceção da Figura 2 (b), todas as curvas apresentaram comportamento semelhante, com pontos de concentração praticamente nula no início do experimento, ponto de ruptura e o aumento gradual da concentração dos íons até o estado de equilíbrio. A partir dos gráficos, foram calculados os parâmetros de eficiência dispostos na Tabela 1 . Como a vazão de $3 \mathrm{~mL} / \mathrm{min}$ atingiu o ponto de ruptura, ou seja, ponto no qual a concentração de saída é igual a $5 \%$ da concentração inicial, antes da coleta da primeira amostra, não foi possível calcular a quantidade útil adsorvida e a ZTM do leito.

Tabela 1 - Parâmetros de eficiência obtidos a partir das curvas de ruptura.

\begin{tabular}{ccccccccc}
\hline & \multicolumn{2}{c}{$1 \mathrm{~mL} / \mathrm{min}$} & \multicolumn{2}{c}{$3 \mathrm{~mL} / \mathrm{min}$} & \multicolumn{2}{c}{$5 \mathrm{~mL} / \mathrm{min}$} & \multicolumn{2}{c}{$7 \mathrm{~mL} / \mathrm{min}$} \\
\cline { 2 - 9 } Parâmetro & Cobre & Zinco & Cobre & Zinco & Cobre & Zinco & Cobre & Zinco \\
& & & & & & & & \\
\hline $\mathrm{q}_{\mathrm{u}}(\mathrm{mmol} / \mathrm{g})$ & 0,1837 & 0,1396 & - & - & 0,0137 & 0,0093 & 0,231 & 0,0420 \\
$\mathrm{q}_{\mathrm{t}}(\mathrm{mmol} / \mathrm{g})$ & 0,4578 & 0,5008 & 0,0902 & 0,1612 & 0,6049 & 0,5396 & 0,1938 & 0,4151 \\
$\mathrm{ZTM}$ & 8,981 & 10,819 & - & - & 14,660 & 14,742 & 13,209 & 13,483 \\
$\% \mathrm{R}_{\mathrm{L}}$ & 60,33 & 70,55 & 17,58 & 26,80 & 45,19 & 44,72 & 32,37 & 31,27 \\
\hline
\end{tabular}


Figura 2 - Curvas de ruptura em sistemas bicomponentes de $\mathrm{Cu}^{2+}$ e $\mathrm{Zn}^{2+}$ a (a) 1 $\mathrm{mL} / \mathrm{min}$, (b) $3 \mathrm{~mL} / \mathrm{min}$, (c) $5 \mathrm{~mL} / \mathrm{min} \mathrm{e} \mathrm{(d)} 7 \mathrm{~mL} / \mathrm{min}$.

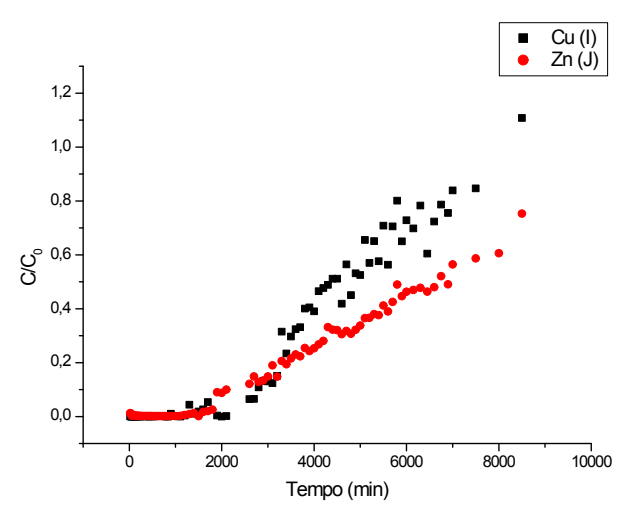

(a)

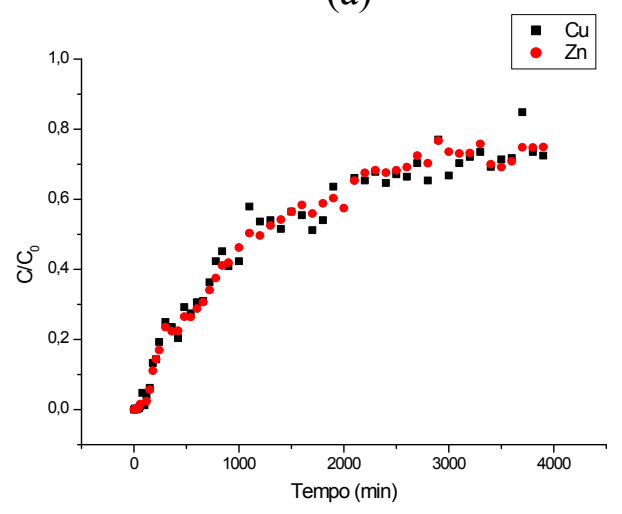

(c)

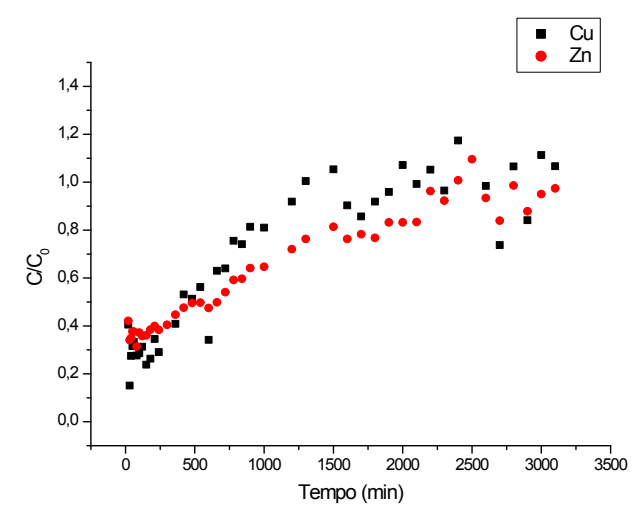

(b)

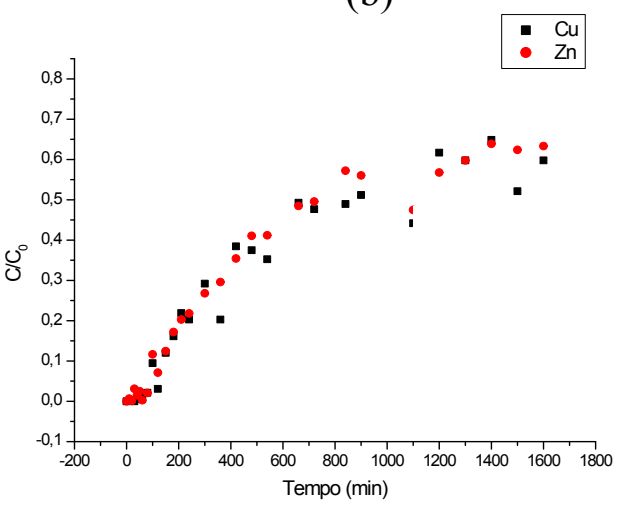

(d)

Estudos multicomponentes são de fundamental importância, pois se aproximam mais do processo real de tratamento de efluentes. Na adsorção em sistemas multicompostos, geralmente ocorre competição dos adsorbatos pelos sítios ativos do adsorvente. Tal competição é gerida por diversos fatores físico-químicos dos metais e peculiaridades da argila que tornam mais complexa a predição dos resultados. No estudo de vazão apresentado, notase uma diferença significativa entre as curvas de ruptura das Figuras 3 (a) e 3 (b), nas quais os íons de cobre se mostram melhor adsorvidos. Já nas Figuras 3 (c) e 3 (d), as curvas não demonstram nenhuma diferença de remoção significativa entre os dois metais. Entretanto, quando analisados os parâmetros de eficiência, novamente comprova-se a melhor seletividade dos íons cobre, uma vez que esse metal apresenta valores inferiores de ZTM.

O resultado obtido concorda com estudo prévio utilizando a mesma argila em sistemas estáticos de adsorção (Almeida et al., 2014). Contudo, ambos divergem de estudo com soluções monocompostas, no qual a vermiculita apresentou maior afinidade pelos íons $\mathrm{Zn}^{2+}$ do que por íons $\mathrm{Cu}^{2+}$ (Nishiwaka et al., 2012 a). Segundo Padilha-Ortega et al. (2013), a sequência de afinidade metálica em um adsorvente depende da massa específica e do raio iônico dos adsorbatos. Todavia, os valores dessas propriedades do cobre e do zinco são bastante próximos e provavelmente não interferem na seletividade. A velocidade de dissociação do íon com as moléculas de água, entretanto, pode justificar a melhor seletividade do cobre em detrimento da maior afinidade do zinco. Íons de cobre exibem a mais rápida taxa 
de troca com a água, podendo de desligar das moléculas de água e estabelecer ligações com a vermiculita antes do que os íons $\mathrm{Zn}^{2+}$ (Helm e Merbach, 2005). Sendo assim, o cobre ocupa os poros da argila primeiro e é mais adsorvido nos sistemas binários.

A vazão de $1 \mathrm{~mL} / \mathrm{min}$ apresentou os menores valores de $\mathrm{ZTM}$ e $\% \mathrm{R}_{\mathrm{L}}$, revelando-se a vazão ótima de operação do sistema. Entretanto, ela demanda um tempo de ensaio muito maior do que as demais vazões, acarretando em maiores erros experimentais.

\section{CONCLUSÕES}

O estudo fluidodinâmico em leito fixo comprovou a competitividade dos íons $\mathrm{Cu}$ (II) e $\mathrm{Zn}$ (II) pelos sítios ativos da vermiculita expandida, concordando com resultados obtidos por Almeida et al (2014) em experimentos binários estáticos de cinéticas e isotermas. Embora a vermiculita possua maior afinidade pelos cátions de zinco em soluções monocompostas (Nishiwaka et al., 2013), íons cobre apresentam melhor seletividade em misturas contendo os dois metais. A inversão está relacionada com a propriedade dos cátions de cobre de se desligarem das moléculas de água do complexo octaédrico e se ligarem aos sítios da vermiculita mais rapidamente do que os cátions de zinco, sendo adsorvidos primeiro.

De acordo com a especiação metálica, a faixa de $\mathrm{pH}$ adequada para que não ocorresse precipitação química foi de até 4,5 . A vazão ótima de operação foi de $1 \mathrm{~mL} / \mathrm{min}$, com valores de ZTM e \%RL, respectivamente, 8,981 e 60,33 para o cobre e 10,819 e 70,55 para o zinco.

\section{REFERÊNCIAS}

ALEXANDRE-FRANCO, M.; ALBARRÁN-LISO, A.; GÓMEZ-SERRANO, V. An identification study of vermiculites and micas. Adsorption of metal ions in aqueous solution. Fuel Proc. Tech., v. 92, p. 200-205, 2011.

ALMEIDA, H. J., ALMEIDA NETO, A. F., VIEIRA, M. G. A., Competitive adsorption kinetics and equilibrium of zinc and copper ions in expanded vermiculite. $X$ Encontro Brasileiro de Adsorção - EBA, 2014.

ALMEIDA NETO, A. F. Caracterização e avaliação de argilas como adsorventes na remoção e eluição de íons cobre e mercúrio em diferentes sistemas. Campinas: Faculdade de Engenharia Química, Universidade Estadual de Campinas, 2011. Dissertação (Doutorado).

BHATTACHARYA, A. K.; MANDAL, S. N.; DAS, S. K. Adsorption of Zn(II) from aqueous solution by using different adsorbents. Chem. Eng. J., v.123, p.43, 2006.

CHUI, Q. S. H. Uso de vermiculita massapé paulistana como adsorvedora de metais. Eng. Sanit. Ambient., v.10, p.58-59, 2005.

CONAMA - Conselho de Meio Ambiente. Resolução n 430, 2011.

DERÍSIO, J.C. Introdução ao Controle de Poluição Ambiental. Sigmus, CETESB, 3 a Edição, 2005.

FONSECA, M. G., DE OLIVEIRA, M. M., ARAKAKI, L. N. H., ESPINOLA, J. G. P., AIROLDI, C. Natural vermiculite as an exchanger support for heavy cations in aqueous solution. J. of Col. and Inter. Sci., v. 285, p. 50-55, 2005. 
FRANCO, A. F.; ALBARRÁN-LISO, A.; GÓMEZ-SERRANO, V. An identification study of vermiculites and micas. Adsorption of metal ions in aqueous solution. Espanha: Universidade de Extremadura, 2010.

GEANKOPLIS, C. J. Transport Process and Unit Operations. EUA: PTR Pretice Hall, $3^{\mathrm{a}}$ edição, p. 703-704, 1993.

GRIM, R.F. Clay Mineralogy. Nova Iorque: McGraw Hill Book Company, 2a edição, 1968.

HELM, L.; MERBACH, A. E. Inorganic and Bioinorganic Solvent Exchange Mechanisms. Chem. Rev., v. 105, p. 1923-1959, 2005.

MACHADO, L. C. R.; TORCHIA, C. B; LAGO, R. M. Floating photocatalysts based on $\mathrm{TiO} 2$ supported on high surface area exfoliated vermiculite for water decontamination. Catal. Com., v.7, p.538-539, 2006.

MOHAN, D.; PITTMAN, C. U.; STEELE, P. H. Single binary and multi-component adsorption of copper and cadmium from aqueous solution on Kraft ligmin - a biosorbent. J. Colloid Interf. Sci., v. 297, p. 489-504, 2006.

NISHIKAWA, E., ALMEIDA NETO, A. F., VIEIRA, M. G. A. Avaliação de vermiculita expandida como adsorvente na remoção de íons de zinco. XXXIII Cong. Inter. de Eng. Sanit. e Amb.- XXXIII AIDIS, p.1 - 9, 2012 (a).

NISHIKAWA, E., ALMEIDA NETO, A. F., VIEIRA, M. G. A. Equilibrium and Thermodynamic Studies of Zinc Adsorption on Expanded Vermiculite. Ads. Scien. \& Tech., v.30, p.759-772, 2012 (b).

PADILHA-ORTEGA, E.; RAMOS, R. L.; CANO, J. V. F. Binary adsorption of heavy metals from aqueous solutions onto natural clays. Chem. Eng. J., v. 225, p. 535-546, 2013.

PERIĆ, J.; TRGO, M.; MEDVIDOVIĆ, N. V., Removal of zinc, copper and lead by natural zeolite - a comparison of adsorption isotherms. Water Research, v.38, p.1893-1899, 2004.

UGARTE, J. F. O.; MONTE, M. B. M. Estudo da vermiculita como adsorvente de óleo e metal pesado. Rio de Janeiro: CETEM, 2006.

\section{AGRADECIMENTOS}

Os autores agradecem o CNPq e o SAE/Unicamp pelo apoio financeiro. 